\title{
Caracterización de compositos biocerámicos en esponjas de titanio mediante interpenetración por corriente pulsada asistida
}

\author{
Nathyely Marisol León-Lugo ${ }^{1}$, Sergio Alberto Torres-Vázquez² \\ Juan José Cortes-Molina ${ }^{3}$
}

\author{
${ }^{1}$ Procesos Industriales, ICA Instituto Politécnico Nacional, Av. Luís Enrique Erro, Edificio 2 PB. Unidad Profesional Adolfo \\ López Mateos, Zacatenco, Gustavo A. Madero, CP 07738, Ciudad de México, México. e-mail: nleon@ipn.mx \\ ${ }^{2}$ Procesos Industriales, ICA Instituto Politécnico Nacional, Av. Luís Enrique Erro, Edificio 2 PB. Unidad Profesional Adolfo \\ López Mateos, Zacatenco, Gustavo A. Madero, CP 07738, Ciudad de México, México e-mail: lanix@gmail.com \\ ${ }^{3}$ Procesos Industriales, Instituto Politécnico Nacional, CECyT No. 1; Av. 510 No. 1000 Col. Ejidos de Aragón C.P. 07480, \\ Ciudad de México, Distrito Federal, México.e-mail: jcortesm@ipn.mx
}

\section{RESUMEN}

Actualmente, se están llevando a cabo una gran cantidad de estudios sobre el desarrollo tecnológico de los procesos de manufactura para soportes (andamios) de ingeniería de tejidos humanos [1]. La impresión 3D aplicada a la ingeniería de tejidos requiere del desarrollo de técnicas computacionales como el modelado estereolitográfico y de procesos de manufactura especiales como son el sinterizado por láser o la deposición por extrusión, por mencionar sólo algunos [2]. A pesar de los avances, sus aplicaciones se han visto limitadas debido a las características y propiedades de los materiales usados, ya que éstos deben cumplir con la condición de ser biomateriales. Por otra parte, los sustratos para ingeniería de tejidos deben mimetizar, en lo posible, las características del tejido donde se va a aplicar, tanto en sus características biológicas como mecánicas. La arquitectura de estos materiales debe permitir la adhesión, nucleación, crecimiento, proliferación y transporte de células vivas para la regeneración del tejido [3]. Lo anterior muestra la creciente importancia de conseguir andamios porosos [4].

En el presente trabajo se obtuvo titanio con diferentes niveles de porosidad (esponjas metálicas), con la finalidad de generar un tamaño promedio de poro conveniente.

La síntesis de las esponjas metálicas se llevó a cabo mediante sinterizado por corriente pulsada asistida, utilizando un espaciador. El contenido del espaciador modifica el gradiente de temperatura durante la síntesis; sin embargo, se observó que las características morfológicas y cristalinas del material no se modificaron. Durante el estudio, las condiciones durante el sinterizado se mantuvieron constantes y reproducibles. Las propiedades y composición de los materiales obtenidos fueron analizadas y comparadas con las de los materiales base. Los resultados obtenidos sugieren que este material podría ser usado exitosamente en la aplicación clínica.

Palabras clave: biomaterial, esponja de titanio, sinterizado.

\section{ABSTRACT}

Recently, it has been directing some special attention to the development of new manufacturing processes, with engineering applications in human tissues [1]. 3D printing applied to tissue engineering requires pulse computational techniques such as stereolithographic modeling, and special manufacturing processes, to name a few; Laser Sintering or Extrusion Deposition [2]. Despite the advances, applications have been limited due to the characteristics and properties of the materials used, so they must meet the biomaterial conditions. On the other hand, biomimicry of materials should be biologically and mechanically imitated when possible on the aim and surrounding tissue. The architecture of these materials must allow adhesion, nucleation, growth, proliferation and transport of living cells for tissue regeneration [3]. Hence, it is exhibited the importance of getting porous scaffolds [4].

In the present work, titanium was obtained with different levels of porosity (metal sponges), in order to locate an average convenient pore size. 
The synthesis of metal sponges was performed by pulsed current assisted sintering using a spacer. The content of the spacer modifies the temperature gradient during the synthesis; however it was noted that the morphological characteristics of the material and crystal were unchanged. During sintering, the operating conditions were constant and reproducible. The properties and composition of the materials obtained were analyzed and compared to commercial materials. The results suggest that this material could be successfully used in clinical applications.

Keywords: biomaterial, sintered, scaffold, titanium sponge .

\section{INTRODUCCIÓN}

El Titanio es de interés particular para aplicaciones biomédicas debido a su excepcional biocompatibilidad y resistencia a la corrosión, además de bajo peso específico comparado contra otros metales aplicados en ésta área de interés.

De tal manera, es deseable que el Titanio poroso pueda ser utilizado en el remplazo parcial o total de un elemento óseo. Durante la investigación se determinaron las condiciones de síntesis para obtener Titanio con diferentes niveles de porosidad a partir de tecnología de polvo; con el objetivo de comparar sus propiedades mecánicas contra las del Titanio como sólido compacto, mismas que se encuentran en la literatura. Se fabricaron monolitos de Titanio sinterizado por corriente pulsada utilizando la técnica de espaciador, para generar la porosidad. Los monolitos fueron sometidos a ensayos de compresión y nano indentación para determinar las propiedades mecánicas, mismas que se utilizaron para validar los resultados obtenidos en la estimación del módulo de elasticidad y del límite elástico. Los resultados obtenidos fueron discutidos a reserva de aprobar el Titanio poroso para justificar la decisión de utilizar este material como implante. Los resultados sugieren que el material no fallará en las zonas críticas donde se podría llevar a cabo una unión con el sistema muscular.

El Titanio poroso puede tener un sin número de aplicaciones como remplazo óseo. Aun cuando existe evidencia de la biocompatibilidad del Titanio, mediante los resultados de esta investigación se propone la modificación del peso especifico del material para incrementar la biocompatibilidad así como también la bioactividad.

Por otra parte, el procesamiento de materiales por fundición se lleva a cabo a escala industrial y es posible generar grandes bloques de material poroso a partir de un método de síntesis, denominado burbujeo, donde se utiliza un gas inerte para nebulizar el material en estado líquido, una vez obtenido el espacio vacío deseado se solidifica el material. Sin embargo, no es posible controlar la densidad relativa del material, el tamaño de poro ni la distribución de poros lo que impide el control de calidad para la generación de piezas pequeñas; de tal manera que no es un proceso atractivo para generar biomateriales.

Diversos investigadores resaltan las ventajas que tienen los implantes metálicos con porosidad superficial desde un $45 \%$ hasta $65 \%$ ya que la recepción de este tipo de materiales es ligeramente mayor con respecto a los que mantienen menor rugosidad superficial, la porosidad superficial se realiza a fin de incrementar la rugosidad de la superficie para promover la adhesión de células óseas y facilitar un entorno biomimético [5]. Algunas técnicas de síntesis son, deposito electroquímico, deposito físico en fase vapor, deposito químico en fase vapor, pulverización catódica y atomización.

La metalurgia de polvos tiene desarrollados diferentes procesos de obtención de sólidos compactos a partir de polvos, en particular el proceso de sinterizado se aplica a escala industrial para la generación de piezas con geometrías simples y complejas [5,6].

Para producir material de Titanio con porosidad abierta, se utilizó el sinterizado de composite compacto de polvo y material de espaciador, siendo este uno de los métodos más prometedores, ya que puede producir tamaños de poro desde micras hasta milímetros. Para obtener la estructura de poro abierto con las propiedades requeridas, es necesario controlar:

- $\quad$ Propiedades del polvo.

- $\quad$ Propiedades cuerpo verde.

- $\quad$ Proceso de sinterización [6].

Las propiedades del polvo están controladas a partir de la forma de las partículas, tamaño y distribución de tamaño, rugosidad de la superficie e impureza e índice de aglomeración. Es importante señalar que las propiedades del polvo afectan a las propiedades de los materiales porosos tales como la forma de poro, distribución de tamaño, área superficial y por su puesto sus propiedades mecánicas. 
El tamaño y la forma del material que se utiliza como espaciador afecta al tipo de poros producidos. Por ejemplo, se podría dar poros esféricos o angulares con diferentes tamaños de poro. La elección del espaciador debe ser tal que se descomponga químicamente a bajas temperaturas [7]. Una nota importante es evitar la contaminación del polvo tanto como sea posible.

\section{MATERIALES Y MÉTODOS}

Este trabajo se realizó utilizando polvo de Titanio $-325 \mu \mathrm{m}$ mesh, con un tamaño de partícula entre 10 hasta 44 $\mu \mathrm{m}$ y $99.5 \%$ de pureza, disponible comercialmente por Alfa Aesar. El polvo fue caracterizado por rayos X previo a la síntesis, en un equipo D8 Advance Bruker a Cu K- $\alpha(\lambda=1.540598 \AA$ ̊). De igual manera se examinó la morfología de las partículas del polvo a través de microscopía electrónica de barrido en un equipo JEOL JSM-6701F por SE $\left(200 \mathrm{nA}\right.$ a15 kV). Posteriormente el polvo fue secado en un horno a $120^{\circ} \mathrm{C}$ por $2 \mathrm{~h}$.

De acuerdo al difractograma mostrado en la Figura 1 se observa que el polvo de Titanio presenta su fase cristalina hexagonal compacta En la Figura 1 se observa la calidad de material De acuerdo al patrón de difracción el pico más intenso, la orientación preferencial corresponde (011).

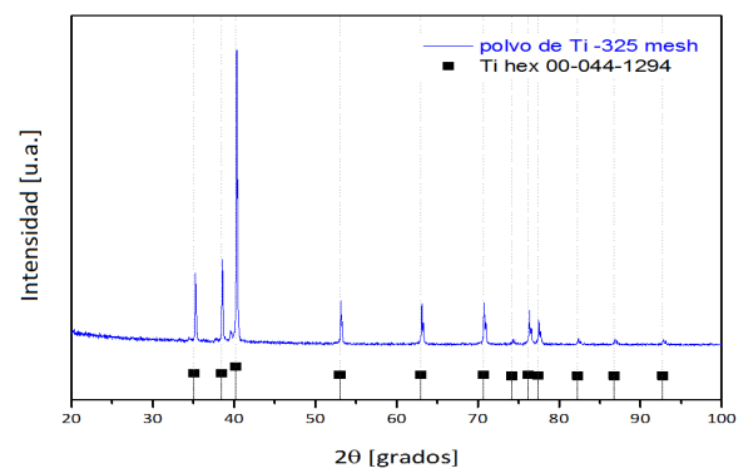

Figura 1: Patrones de difracción del polvo de Ti comparad contra los datos de la carta Ti hex 00-044-1294.

Se verifico la pureza del polvo de Titanio comercial a través de espectroscopia de energía dispersiva (EDS) mediante la cual el análisis indica un $100 \%$ de Titanio en la muestra. De acuerdo a la micrografía de 5000X de la muestra, se observa que las partículas del polvo muestran una morfología irregular en forma detrítica, con lo cual se alude el efecto del proceso de obtención del polvo. En la figura 2 se puede observar el tamaño aproximado de las partículas, siendo este de $20 \mu \mathrm{m}$.

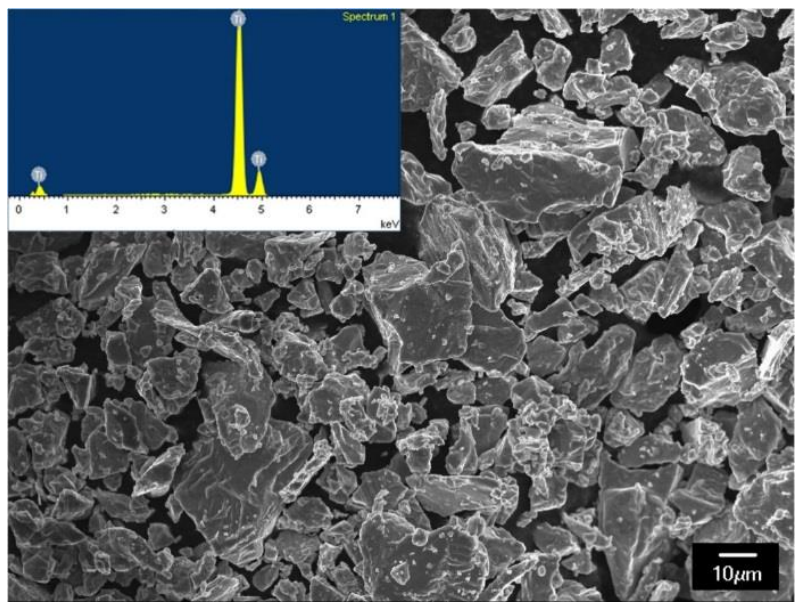


Figura 2: Micrografía del polvo de titanio y su espectrografía por MEB-EDS a 5000X.

\subsection{Cuerpo en verde}

Para producir materiales porosos por el método de sinterización, el polvo es el material inicial. Cuerpo verde es el término para el cuerpo del pre-sinterizado de polvo. El proceso metalúrgico de polvo de sinterizado se le denomina técnica del espaciador [8].

Las etapas del proceso se enumeran como:

- Mezcla del polvo de titanio fino con la sustancia del espaciador

- Compactación del cuerpo verde

- La eliminación del espaciador

La mezcla del material se llevó a cabo de forma homogénea en un molino para mantener una proporción $20 \%, 30 \%$ y $40 \%$ volumen del espaciador con respecto al material base.

La compactación del polvo se realizó a $51 \mathrm{MPa}$, a una temperatura de $700^{\circ} \mathrm{C}$ de forma escalonada, con una velocidad de $100^{\circ} \mathrm{C} / \mathrm{min}$. Posteriormente se procedió a eliminar el espaciador mediante un baño ultrasónico por $2 \mathrm{hrs} \mathrm{a} 60^{\circ} \mathrm{C}$. El volumen considerado para cada una de las muestras fue de $0.5 \mathrm{~cm}^{3}$. En la Figura 3 se muestra cada uno de los componentes de sistema constituidos de grafito.

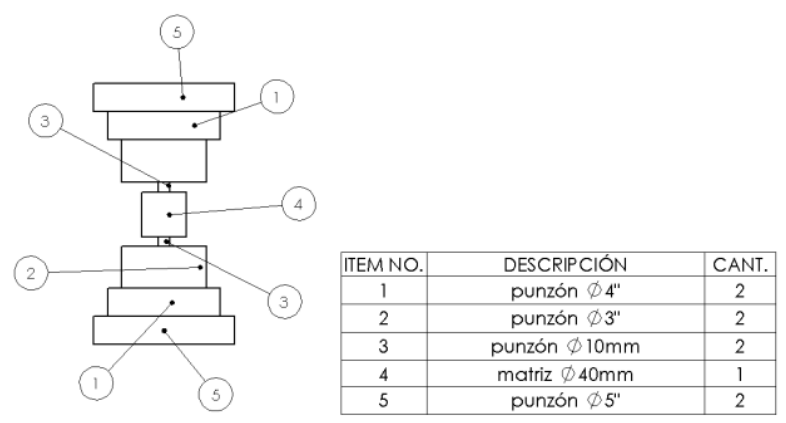

Figura 3: Diagrama de despiece del sistema de sinterizado.

En la Figura 4 se observa el disponible para la muestra de Titanio, así como el diseño de cada uno de los elementos que componen al sistema.
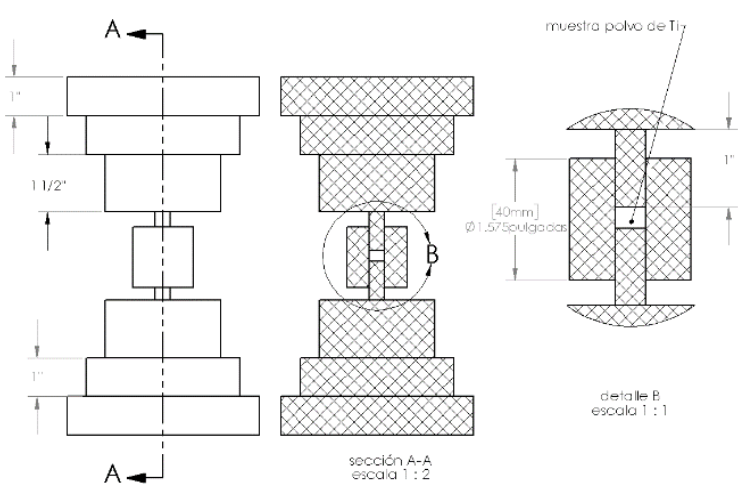

Figura 4: Dibujo del sistema de sinterizado.

\subsection{Sinterización}

El equipo disponible para realizar el sinterizado por corriente pulsada asistida es un Dr. SINTER SPS. En la Ta- 
bla 1 se muestra el valor de las variables controladas automáticamente. La corriente eléctrica fue pulsada (control on/off) de forma constantes $12 / 2$, CD.

Tabla 1: Correlación entre las variables de proceso durante el sinterizado.

\begin{tabular}{|c|c|c|c|}
\hline TIEMPO (MIN) & CORRIENTE (A) & TENSIÓN (V) & TEMPERATURA $\left({ }^{\circ} \mathrm{C}\right)$ \\
\hline 00:00 & 0,00 & 0,00 & 37,00 \\
\hline $00: 30$ & 400,00 & 2,50 & 62,00 \\
\hline 01:00 & 400,00 & 2,50 & 110,00 \\
\hline 01:30 & 600,00 & 2,50 & 155,00 \\
\hline 02:00 & 400,00 & 2,00 & 198,00 \\
\hline $02: 30$ & 400,00 & 2,00 & 202,00 \\
\hline 03:00 & 400,00 & 2,00 & 199,00 \\
\hline $03: 30$ & 400,00 & 2,00 & 200,00 \\
\hline 04:00 & 600,00 & 4,00 & 200,00 \\
\hline 04:30 & 1000,00 & 3,50 & 283,00 \\
\hline 05:00 & 1000,00 & 3,50 & 377,00 \\
\hline 05:30 & 1200,00 & 4,00 & 462,00 \\
\hline 06:00 & 1200,00 & 4,00 & 550,00 \\
\hline 06:30 & 1300,00 & 4,00 & 644,00 \\
\hline 07:00 & 1400,00 & 4,50 & 731,00 \\
\hline $07: 30$ & 1400,00 & 4,50 & 815,00 \\
\hline 08:00 & 1400,00 & 4,50 & 906,00 \\
\hline 08:30 & 1600,00 & 4,50 & 995,00 \\
\hline 09:00 & 1000,00 & 3,50 & 1076,00 \\
\hline 09:30 & 1100,00 & 4,00 & 1079,00 \\
\hline 10:00 & 1200,00 & 3,50 & 1080,00 \\
\hline $10: 30$ & 1200,00 & 3,50 & 1080,00 \\
\hline 11:00 & 1200,00 & 3,50 & 1080,00 \\
\hline $11: 30$ & 1200,00 & 3,50 & 1080,00 \\
\hline $12: 00$ & 1200,00 & 3,50 & 1080,00 \\
\hline
\end{tabular}

\section{RESULTADOS}

Se sintetizaron de manera reproducible un conjunto de piezas con diferente porosidad, a $0 \%, 20 \%, 30 \%$ y $40 \%$.

En la Figura 5 se muestran tres piezas representativas de las esponjas de Titanio con un volumen de $0.5 \mathrm{~cm}^{3}$. El diámetro de los monolitos está en función del diseño del sistema de sinterizado mostrado en la Figura 4. 


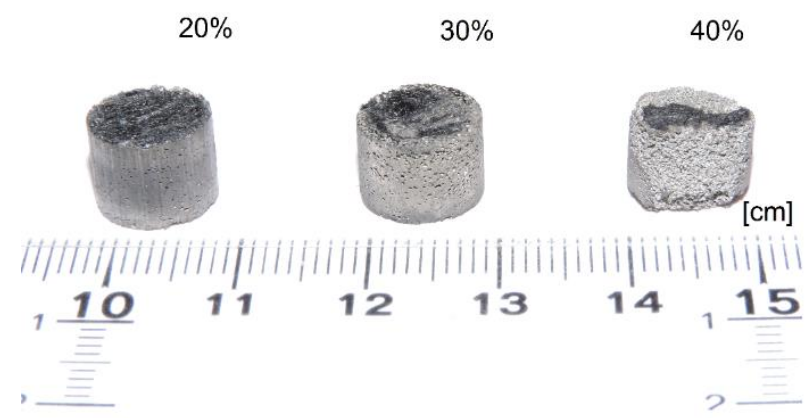

Figura 5: Monolitos de las esponjas de Titanio.

A fin de realizar los ensayos de nano dureza los monolitos fueron cortados de forma axial por láser afín de obtener un espesor de cada disco de $2 \mathrm{~mm}$. Se comparó el material sinterizado contra una placa de Titanio comercial grado 1, 99.998\% de pureza, de 2mm de espesor. En la Figura 6 se muestran los especímenes disponibles para efectuar la evaluación mecánica. Se analizaron 6 especímenes por cada categoría

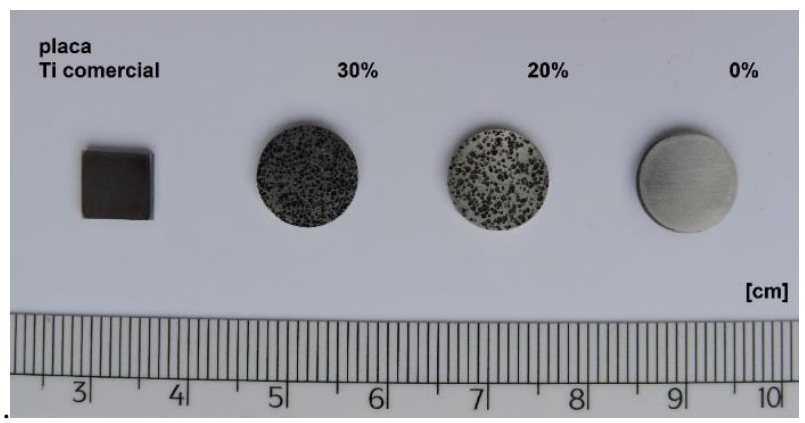

Figura 6: Muestras de Titanio comercial (placa), esponjas de Titanio al 30 y $20 \%$ de porosidad y Titanio compacto. 4

Durante el ensayo de nano dureza, se utilizó un nanoidentador de punta diamante (Figura 7)

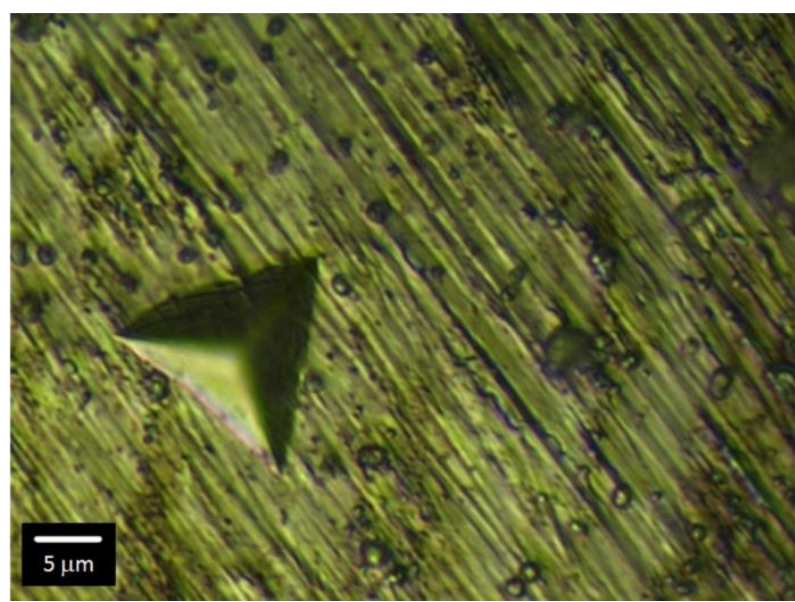

Figura 7: Micrografía de la huella del nano indentador.

\section{DISCUSIÓN}


Al obtener la muestra en verde, se analizó el comportamiento del desplazamiento de los punzones. Se observó que el incremento de corriente y tensión eléctrica no se mantuvo proporcional con respecto a la temperatura; sin embargo, se puede aludir que esta situación se refiere al control automático del equipo (Figura 8). Por otra parte, a mayor porosidad mayor contenido se espaciador se requiere, de tal manera que el coeficiente de compactación es menor a mayor porcentaje del cerámico. El volumen de compactación fue mayor para las muestras de menor porosidad como se observa en la Figura 9.

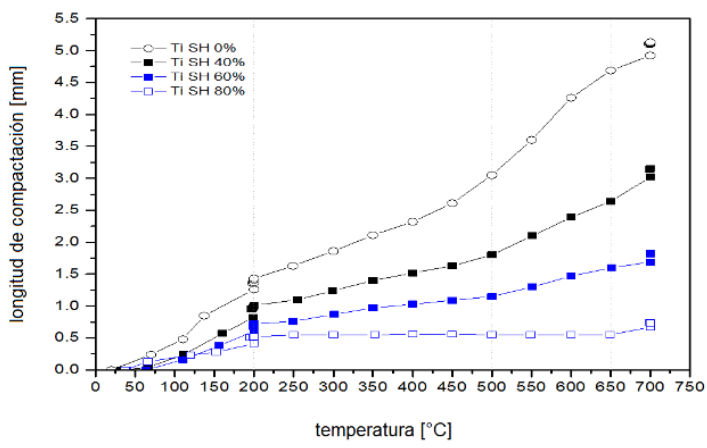

Figura 8: Efecto del coeficiente de expansión con respecto al coeficiente de expansión de la muestra.

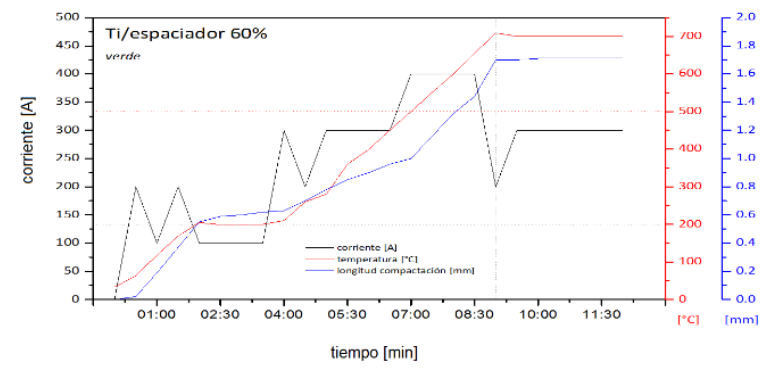

Figura 9: Comportamiento de la corriente eléctrica y nivel de compactación con respecto a la temperatura.

De acuerdo a las etapas de sinterizado, la densificación se completa a los $200^{\circ} \mathrm{C}$ y la sinterización del material a $\operatorname{los} 500^{\circ} \mathrm{C}$, esto se observa en la Figura 10.

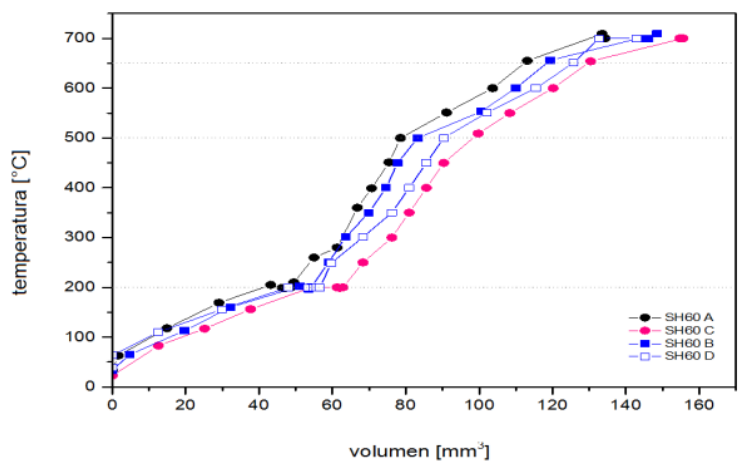


Figura 10: Efecto de la densificación y sinterización sobre el volumen de compactación.

Al sinterizar completamente la muestra se observó que el comportamiento de la corriente no es lineal con respecto a la temperatura como se observa en la Figura 11.

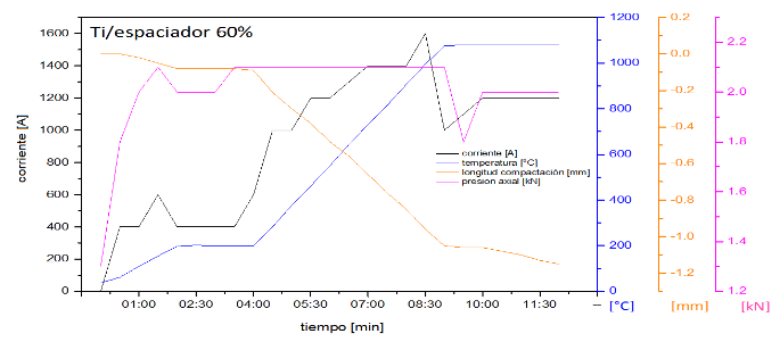

Figura 11: Comportamiento de las variables, corriente eléctrica, nivel de compactación y fuerza axial durante la sinterización.

La dureza del Titanio obtenido por sinterizado por corriente pulsada asistida incrementa con respecto a un Titanio laminado; sin embargo, al incrementar la porosidad del material disminuye la dureza, como se observa en la Figura 12.

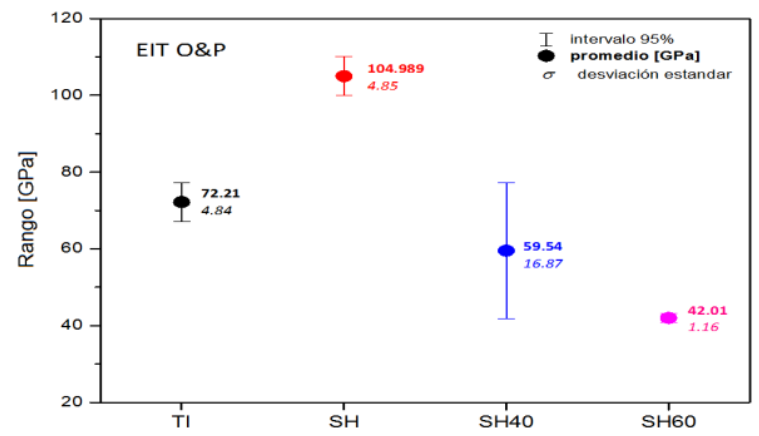

Figura 12: Micrografía de la huella del nanoidentador

\section{CONCLUSIONES}

En consecuencia, se puede indicar que el material fabricado mediante sinterizado por corriente asistida pulsada es repetible, siempre y cuando se determinen las condiciones de síntesis y control de las variables de proceso. Los materiales muestran un incremento en sus propiedades mecánicas mediante esta sinterizado por corriente pulsada asistida y a la vez se pueden crear esponjas metálicas con propiedades semejantes a las del hueso humano.

Con el presente método se puede fabricar esponjas de titanio con características específicas y adecuadas para indicar que puede ser un material prometedor para ser utilizado biomaterial, sustituto de piezas óseas sujetas a carga.

\section{AGRADECIMIENTOS}

Los autores desean agradecer de manera especial al Centro de Investigación e Innovación Tecnológica (CIITEC IPN) por otorgar las facilidades de infraestructura. Al Instituto Politécnico Nacional por el apoyo financiero otorgado al proyecto SIP20161081 y a la Comisión de Operación y Fomento de Actividades Académicas del Instituto (COFAA - IPN). Así como también al Centro de Nanociencias y Micro y Nanotecnologías y a la Escuela Superior de Ingeniería Química e Industrias Extractivas (ESIQIE-IPN) por los análisis de Difracción de Rayos X, Microscopia Electrónica de Barrido y Nanoidentación. 


\section{BIBLIOGRAFÍA}

[1] FALLAHIAREZOUDAR, E., AHMADIPOURROUDPOSHT, M., IDRIS, A., et al, "A review of: application of synthetic scaffold in tissue engineering heart valves", Materials Science \& Engineering C, Materials for Biological Applications, v. 48, pp. 556-565, 2015.

[2] GIANNITELLI, S. M., ACCOTO, D., TROMBETTA, M., et al, "Current trends in the design of scaffolds for computer-aided tissue engineering", Acta Biomaterialia, v. 10, n.2, pp. 580-594, 2014.

[3] TANG, D., TARE, R. S., YANG, L.-Y., et al, "Biofabrication of bone tissue: Approaches, challenges and translation for bone regeneration", Biomaterials, v. 83, pp. 363-382, 2016.

[4] TORRES, Y., LASCANO, S., BRIS, J., et al., "Development of porous titanium for biomedical applications: A comparison between loose sintering and space-holder techniques", Materials Science and Engineering C, v. 37 n.1, pp. 148-155, 2014.

[5] WIRIA, F. E., SHYAN, J. Y. M., LIM, P. N., et al., "Printing of Titanium implant prototype", Materials \& Design, v. 31, pp. S101-S105, 2010.

[6] TUNCER, N., BRAM, M., LAPTEV, A., et al., "Study of metal injection molding of highly porous titanium by physical modeling and direct experiments", Journal of Materials Processing Technology, 2014.

[7] JHA, N., MONDAL, D. P., DUTTA MAJUMDAR, J., et al., "Highly porous open cell Ti-foam using NaCl as temporary space holder through powder metallurgy route", Materials and Design Journal, v. 47, pp. 810-819, 2013.

[8] BYOUNGGAB, L., TAEKYUNG, L., YONGMOON, L., et al., "Space-holder effect on designing pore structure and determining mechanical properties in porous titanium", Materials and Design Journal, v. 57, pp. 712$718,2014$. 